University of Wollongong

Research Online

SMART Infrastructure Facility - Papers

Faculty of Engineering and Information

Sciences

$1-1-2015$

An approach to plan and evaluate the location of radiotherapy services and its application in the New South Wales, Australia

Nagesh Shukla

University of Wollongong, nshukla@uow.edu.au

Rohan Wickramasuriya

University of Wollongong, rohan@uow.edu.au

Andrew Alexis Miller

University of Wollongong, amiller@uow.edu.au

Pascal Perez

University of Wollongong, pascal@uow.edu.au

Follow this and additional works at: https://ro.uow.edu.au/smartpapers

Part of the Engineering Commons, and the Physical Sciences and Mathematics Commons

Research Online is the open access institutional repository for the University of Wollongong. For further information contact the UOW Library: research-pubs@uow.edu.au 


\section{An approach to plan and evaluate the location of radiotherapy services and its application in the New South Wales, Australia}

\section{Keywords}

plan, approach, evaluate, application, its, location, services, south, australia, wales, radiotherapy

Disciplines

Engineering | Physical Sciences and Mathematics

\section{Publication Details}

Shukla, N., Wickramasuriya, R., Miller, A. Alexis. \& Perez, P. (2015). An approach to plan and evaluate the location of radiotherapy services and its application in the New South Wales, Australia. Computer Methods and Programs in Biomedicine, Online First 1-24. 


\title{
An approach to plan and evaluate the location of radiotherapy services and its application in the New South Wales, Australia
}

\author{
Nagesh Shukla $^{1 *}$, Rohan Wickramasuriya ${ }^{1}$, Andrew Miller ${ }^{1,2}$, Pascal Perez ${ }^{1}$ \\ ${ }^{1}$ SMART Infrastructure Facility, Engineering and Information Sciences, University of Wollongong, \\ Wollongong, NSW 2522, Australia \\ ${ }^{2}$ Illawarra Cancer Care Centre, Wollongong Hospital, Wollongong NSW 2500 Australia \\ *Corresponding Author Email: nshukla@uow.edu.au
}

\begin{abstract}
This paper proposes an integrated modelling approach for location planning of radiotherapy treatment services based on cancer incidence and road network-based accessibility. Previous research efforts have established travel distance/time barriers as a key factor affecting access to cancer treatment services, as well as epidemiological studies have shown that cancer incidence rates vary with population demography. Our study is buil on the evidence that the travel distances to treatment centres and demographic profiles of the accessible regions greatly influence the uptake of cancer radiotherapy (RT) services. An integrated service planning approach that combines spatially-explicit cancer incidence projections, and the placement of new RT services based on road network based accessibility measures have never been attempted. This research presents a novel approach for the location planning of RT services, and demonstrates its viability by modelling cancer incidence rates for different age-sex groups in New South Wales, Australia based on observed cancer incidence trends; and estimations of the road network-based access to current NSW treatment centres. Using three indices (General Efficiency, Service Availability and Equity), we show how the best location for a new RT centre may be chosen when there are multiple competing locations.
\end{abstract}

Keywords: radiotherapy services, cancer, location planning, accessibility

\section{Introduction}

Cancer control is a health priority. Cancer is estimated to be the leading cause of burden of disease in Australia in 2010, accounting for 19\% of the total burden, and has a major impact on the Australian community, since one in three men and one in four women in Australia will 
be diagnosed with cancer by the age of 75. By age 85, the risk increases to one in two for men and one in three for women (Cancer Council Australia, 2012). The proportion of elderly people in the population will steadily increase over the next decades due to increased life expectancy (ROTC, 2012a) and the 'baby boomers' ageing population is entering the high incidence period, thereby increasing the number of cancer cases.

Beyond demographic influences, other factors like socio-economic status and ethnicity have also an effect on cancer incidence (ROTC, 2012a) along with geographical variations in the rate of treatment and survival from cancer (Coory et al., 2013; Yu et al., 2014). As the number and diversity of cancer cases increase, the pressure on specialised treatment services will increase, calling for better planning and allocation of healthcare resources, particularly at the regional level.

Radiotherapy (RT) is an essential cancer therapy whether aimed at cure or palliation. The Collaboration for Cancer Outcomes Research and Evaluation (CCORE) literature-based findings were used by the Radiation Oncology Reform Implementation Committee (RORIC) to estimate that $52.3 \%$ of all diagnosed cancer cases in Australia would benefit from radiotherapy at some point after diagnosis. The Australian state health department uses this estimate for planning their RT services (Delaney et al., 2003; ROTC 2012b; Morgan 1999). Radiotherapy is considered to be most cost effective than surgery and chemotherapy, when all costs across the life cycle is considered (Ploquin and Dunscombe 2008). According to the RANZCR, radiotherapy provides similar benefit with lower costs for cancer patient treatment for cures. RT generally costs about 6 per cent of each health dollar spent fighting cancer, but it is a vital part of curing about 40 per cent of all cured cancers. As radiation therapy is provided as an outpatient treatment service, the overall treatment costs are less than other treatments. In terms of effectiveness, an Australian study stated that external-beam radiation therapy is at least as effective as modern Australian surgical techniques (Wilcox et al. 2015). It states that men with localised prostate cancer $(\approx 30.0 \%$ of all new cancers in men) who are treated with external-beam radiation therapy have a cure rate of $95.5 \%$ for intermediate-risk prostate cancer and $91.3 \%$ for high-risk prostate cancer. Also, for breast cancer $(\approx 28 \%$ of all new cancers in women) treatment studies conducted in Canada and Denmark, have shown a 9\%-10\% improvement in overall survival at 10 years for patients that received radiotherapy compared with patients who did not receive radiotherapy (Vinh-Hung and Verschraegen 2004, Ragaz et al. 1997). Like these there are other studies which show better outcomes and cost-effectiveness for RT treatments for different types of cancer and their stages. 
However, measured access to RT services has established that utilization rates are well below this optimal number (Mackillop et al., 1997; Barton 2000; Einhorn et al. 1996; Athas et al., 2000) clearly demonstrating the existence of barriers to access existing RT services. Currently, only 38\% of cancer sufferers receive radiotherapy during their disease journey (ROTC 2012a, Barton and Delaney 2011; Denham 1995) indicating that $\sim 14 \%$ of cancer sufferers, miss the benefit from RT services that may improved cancer control and appropriate evidence-based management (ROTC 2012a, Barton and Delaney 2011). Since the proximity of RT facilities to home has been identified as a major factor enhancing accessibility to and utilization of RT services (Mackillop et al., 1997; Denham, 1995), it is likely that remote and rural patients are facing limited accessibility to radiotherapy on the basis of inadequate transportation and lengthy travel. A literature review has highlighted travel as a perceived barrier to cancer treatment (Payne et al., 2000). Several national and international studies supported the relation between travel distance from radiotherapy centres and uptake rates (The Allen Consulting Group, 2012; Delaney et al., 2005; Williams 2009; Cancer Care Ontario, 2008; Schroen et al. 2005; Craft et al., 2010; Badde et al., 2011; Wigg and Morgan, 2001; Morgan et al., 2010). Although the configuration of centralised networks may be driven by resource efficiency savings, it has reflecting clinical need in rural and remote areas will appear less resource efficient (Asthana et al 2003).

Various studies have examined the effect of geographical accessibility, based on travel times/distances as proxy to travel effort, on uptake of RT based cancer treatment. For example, Madelaine et al. (2002) reported lower treatment rates for rural lung cancer patients in France. Punglia et al. (2006) found that increasing distance to the nearest radiotherapy centre was associated with a decreasing likelihood of receiving post-mastectomy radiation therapy. Greenberg et al., (1988) asserted that lung cancer patients living at greater straight line distance from a specialist cancer centre, in rural USA, were significantly more likely to undergo surgery but were less likely to receive radiotherapy or chemotherapy than closer patients. Athas et al. (2000) also found that breast cancer patients living further than 75 miles from a radiotherapy services centre were significantly less likely to receive radiotherapy than those living closer. It is possible that the detriment of transportation may be even more pronounced in patients who are faced with weeks of daily outpatient treatment, as is common for radiation therapy. Some recent studies have reported the location assessment for public healthcare facilities in US. Batta et al. (2014) has used p-maxian model considering dispersion, population, and equity for obtaining the optimal locations of public facilities. 
Another study by Burkey et al. (2012) has used location-based comparisons based on efficiency and equity to compare healthcare services in four US states. Our paper will use some of these indices (general efficiency, service availability and distance-based equity) to evaluate the potential RT service locations.

Recently, Gabriel et al. (2012) presented the results from the data linkage study for radiotherapy utilisation rates in NSW and Australian Capital Territory (ACT) for years 200406. They concluded that the radiotherapy utilisation rates decreased with increasing distance from patient's residence to the nearest RT facility $(p<0.0001)$. The study quantified RT rates ranging from $27 \%$ for patient living within $50 \mathrm{~km}$ of RT facility to $19 \%$ for patients living more than $400 \mathrm{~km}$ from the nearest RT facility. Recently, various studies (Shukla et al (2014); Shukla et al. (2014); Tyagi et al. (2015)) have only focussed on within organisation (or hospital) level process improvements but have not considered patient accessibility measures for improvements.

The planning of efficient and accessible RT services for cancer care at regional level requires estimates of current and future cancer demand based on the spatial distribution and evolution of various socio-demographic groups, spatial accessibility based on transport network and probabilities of re-treatment. In this study, we will develop a modelling tool which can be systematically used for planning of radiotherapy services.

After detailing the data and methods used in the modelling effort, we will demonstrate how we have applied these modelling methods to plan and evaluate RT services in NSW.

\section{Approach for planning of radiotherapy services}

The proposed approach for modelling and predicting the future cancer incidences and their accessibility to existing RT centres in the state is visualised in Figure 1, and involves datasets such as Australian Institute of Health and Welfare (AIHW) cancer incidence data, Australian Bureau of Statistics (ABS) population projection dataset, $1 \mathrm{~km}$ Australian population grid dataset, road network dataset, and data about the existing radiotherapy (RT) centres.

\subsection{Datasets used in the proposed approach}

\section{A. AIHW cancer incidence dataset}

The AIHW cancer incidence dataset (AIHW, 2014) provides the information about age group and sex specific cancer rates for all and specific cancer types in Australia. The major source 
of this data comes from the Australian Cancer Database (ACD) which contains records of all the primary, malignant cancers (except basal cell and squamous cell carcinomas of the skin) diagnosed in Australia since 1982. The data from ACD is generally used to report national cancer statistics such as incidence, trends, projections, survival, and prevalence. The ACD data is compiled from the data provided by the state and territory cancer registries through Australasian Association of Cancer Registries (AACR). The age-specific rates (ASR) for cancer in males and females, in past years, are used to model and predict the cancer incidences in the state.

\section{B. ABS population projection dataset}

The population projection data prepared by the ABS to permit the Australian Government Department of Social Services to plan and evaluate aged care. These projections are based on the past trends (over a decade) of fertility, mortality, and migration trends, using the cohortcomponent method, where the base population is projected into the future year annually by estimating the effect of births, deaths and migration within each age-sex group according to the specified fertility, mortality and migration assumptions. This datasets contains the age group by sex population numbers for each Statistical Local Area. SLA is an Australian Standard Geographical Classification (ASGC) defined area that covers a Local Government Area (LGA) or part thereof. The median population of SLAs is about 21,000. The population projections cover the period from 2012 to 2026, using the 2011 ABS census year as a base. More information about this dataset is present elsewhere (DoH 2013).

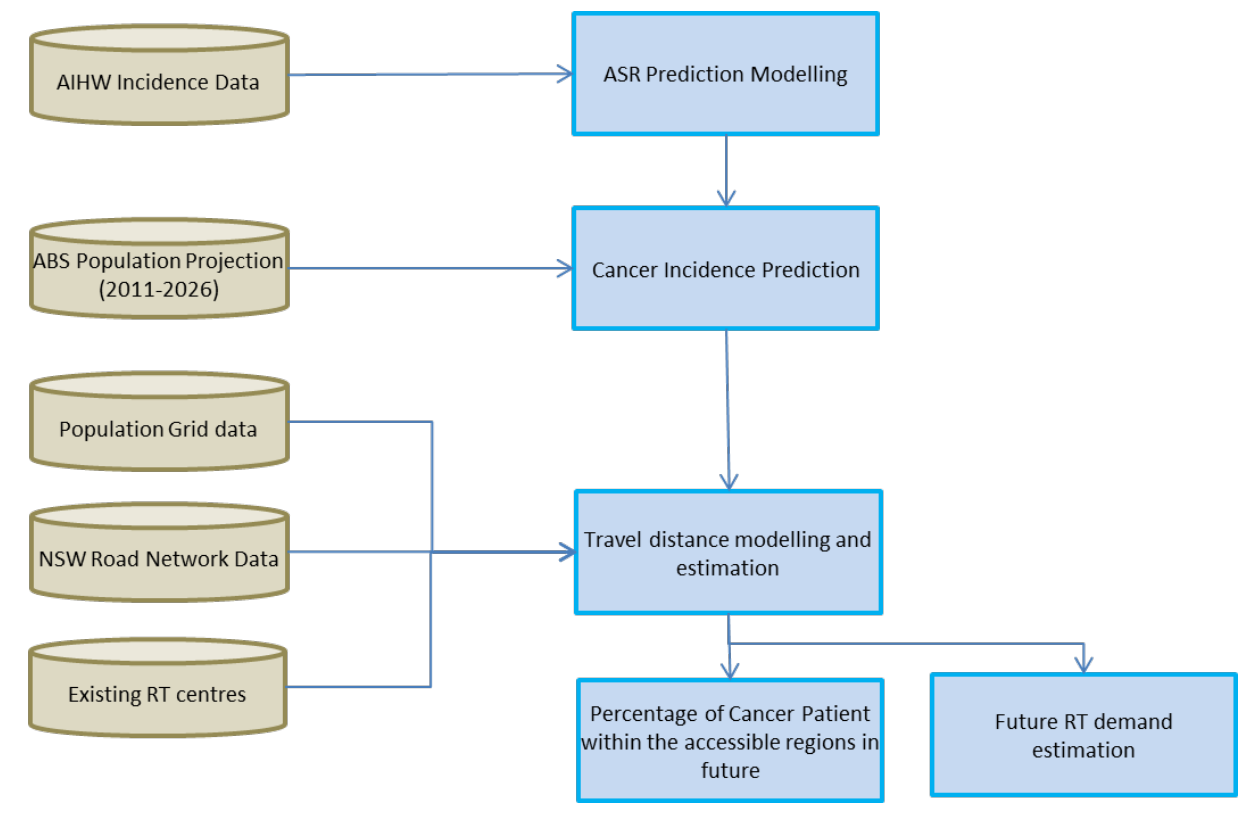


Figure 1: Methodological approach to determine the accessibility of cancer patients to radiotherapy centres

C. Australian Population Grid dataset from ABS Australian Bureau of Statistics (ABS) has released a $1 \mathrm{~km}$ population grid covering the entire country based on the Census data collected in 2011. This dataset represents the finest resolution digital population data available at the national scale According to ABS, this dataset has key advantages of (a) greater spatial accuracy in both urban and rural areas, and (b) the ability to efficiently integrate with other spatial datasets such environmental data. This dataset is used in travel distance modelling to calculate the population coverage.

\section{Road network dataset}

The road network dataset was downloaded from the most up-to-date OpenStreetMap (OSM) database, using Quantum GIS software (Gray, 2008). OpenStreetMap is a crowd-sourced initiative to collect and map roads, trails, and points of interest, with an ultimate aim of building a geographic database that contains every single feature on the planet (Bennett, 2010). OSM data was selected for this study as it is a readily available and open data source with sufficient positional accuracy (Haklay, 2010).

\section{E. Existing radiotherapy (RT) centres}

The data about the existing RT treatment facilities is accessed from Department of Health, Australia. DoH (2014) provides publically available information about radiation therapy services including accommodation and travel schemes, facility locations and treatment options. The facility locations for RT centres were used for estimating the RT treatment accessible regions for future cancer incidences.

\subsection{Methods}

\section{A. Age specific rate (ASR) for cancer incidence modelling}

A non-linear polynomial regression model (of degree 3) was used to model the past trend of the cancer incidences. The polynomial regression models have been developed for each agesex group for male and female. Age specific rates (ASR) from AIHW dataset, for cancer incidences for years 1982 to 2009, have been used to estimate the coefficients of the regression model. The model used for ASR regression is:

$$
A S R_{t}=\beta_{0}+\beta_{1} \times t+\beta_{2} \times t^{2}++\beta_{3} \times t^{3}+\varepsilon_{t}
$$


Where, $A S R_{t}$ is the age-specific rate for a particular age-sex group observed in year $t . \varepsilon_{t}$ is assumed to be independent and identically distributed normal random variable with mean zero and a constant variance.

The AIHW cancer incidence dataset for years 1982-2009 is used for estimating coefficients $\beta_{0}-\beta_{3}$. The confidence level is set to be $95 \%$ for the two sided hypothesis test. If the ASR model for an age-sex model is found significant, then $A S R_{t}$ is estimated based on Eq. (1). Otherwise, mean of $A S R_{t}$ (where, $t=2000,2001, \ldots, 2009$ ) for recent 10 years for a particular age-sex group is used for prediction. In this modelling, we have made following assumptions:

i. this modelling assumes that the incidence is homogeneous across different local government areas (LGAs)

ii. ages were grouped in 5 year interval for this modelling and it assumes that each age group is homogeneous

iii. it is assumed that the past trends in cancer incidences will continue in future

\section{B. Predicting the cancer cases}

Once the regression models for the cancer incidence is obtained for each age-sex group, it is then used together with the ABS population projections dataset to estimate the future cancer cases in the study area. The ABS population dataset is aggregated into a contingency table having attributes such as age group, sex, geographical area, and number of people at risk. This data table is then used together with the predicted ASR for each year (derived from section 2.2.A) to produce cancer cases in different age-sex group in different LGAs. The result of this analysis provides cancer cases distributed in various LGAs in future. Once the number of cancer cases for future years are identified, it can be used to estimate the future radiotherapy demand/workload.

\section{Travel distance modelling and the estimation of population coverage}

Following the literature that supports a strong relationship between travel distance and uptake rates (as discussed in Section 1) we calculated driving distance polygons for all treatment centres using the popular open source software 'pgRouting' (pgRouting Contributors, 2013). Routing functions provided by pgRouting are implemented as an extension to the open source PostgreSQL/PostGIS geospatial database (Douglas and Douglas, 2005). We were mainly 
interested in the function 'pgr_drivingDistance' to calculate driving distance polygons (pgRouting Contributors, 2013). This function computes the cost (distance) to reach every node in the network from a given starting node based on a Dijkstra shortest path solution. Further details of the Dijkstra algorithm and its applications to road networks can be found in Zhan and Noon (1998).

Firstly, the OSM road network of NSW was imported into PostgreSQL/PostGIS as a pgRouting-enabled (i.e. routable) network dataset. A custom SQL function that leverages the default pgRouting function 'pgr_drivingDistance' was written to loop through all treatment centres. This custom function takes each treatment centre as the starting node, and computes all nodes in the road network that can be reached within a specified threshold distance. The concave polygon that encompasses the collection of nodes computed above was then generated using a standard function available in PostGIS. This concave polygon is termed the ‘constant driving distance polygon’ or isochrone.

Considering the fact that dwellings are not uniformly distributed in space, the digital dataset of the population grid was used to estimate the fraction of the population that lives in a particular LGA within a given distance ring.

Let $P_{i, j}^{p}$ be the portion of population of the $i^{\text {th }}$ LGA that lives within the $j^{\text {th }}$ distance ring, and $P_{i}^{t}$ be the total population of the $i^{\text {th }}$ LGA, then the fraction of population (of $i^{\text {th }}$ LGA) in $j^{\text {th }}$ distance ring (denoted by $F R A_{i, j}$ ) is given by:

$$
F R A_{i, j}=\frac{P_{i, j}^{p}}{P_{i}^{t}}
$$

under the condition $\sum F R A_{i, j}=1$ for each LGA.

\section{Radiotherapy based treatment demand estimation}

The predicted cancer cases and distance-based LGA ratios $\left(F R A_{i, j}\right)$ were used together with the radiotherapy rates to estimate the future demand for RT services. The RT rates were chosen based on the distance bands provided in Gabriel et al. (2012). They calculated the actual radiotherapy treatment rates for cancer patients in NSW \& ACT (2004-06) from patient treatment records to estimate the effects of geographic variations on the RT rates. The road distance between patient residence and the nearest RT centre was estimated to compute the rates of radiotherapy for patients living $<50 \mathrm{Km}$, $50-99 \mathrm{Km}, 100-149 \mathrm{Km}, 150-199 \mathrm{Km}$, 200-249 Km, 250-299 Km, 300-349 Km, 350-399, and 400+ Km. The dataset used for this 
study was from 2004-2006. Since this dataset is nearly 10 years old, we have added another data source, i.e., local health district cancer treatment data containing radiotherapy data for years 2006 -2012. This local health district is out of the eight in NSW. Using both of these datasets we have estimated the average radiotherapy rates for cancer patients based on their driving distances to the nearest RT facilities. The combined average rates for RT are presented in Table 1.

Table 1: Radiotherapy rates based on driving distances

\begin{tabular}{|l|c|}
\hline Distance & Average rates \\
\hline$<50 \mathrm{KM}$ & 0.339825 \\
\hline $50-100 \mathrm{KM}$ & 0.30606 \\
\hline $100-150 \mathrm{KM}$ & 0.268176 \\
\hline $150-200 \mathrm{KM}$ & 0.24006 \\
\hline $201-250 \mathrm{KM}$ & 0.2276 \\
\hline $251-300 \mathrm{KM}$ & 0.214825 \\
\hline $301-350 \mathrm{KM}$ & 0.216063 \\
\hline $351-400 \mathrm{KM}$ & 0.18525 \\
\hline$>400 \mathrm{KM}$ & 0.161785 \\
\hline
\end{tabular}

Delaney et al. (2005) have summarised the overall optimal radiotherapy utilization rate for individual cancers such as skin, breast, lung, gastrointestinal, genitourinary and others. Evidence for radiotherapy based treatments such as systematic review of relevant randomised studies, randomised/controlled trials, and case series, were used in modelling decision trees for determining optimal radiotherapy rates. For more information about these rates and methodology see Delaney (2007). The optimal RT rates varied from 0\% in case of liver cancer patients and 92\% for patient with central nervous system tumors. The optimal radiotherapy utilisation rate was estimated to be $52.3 \%$ of all the notifiable cancer in Australia. The SSDB (2012) report indicates that an additional 23\% of the cancer patients receive at least 2 course of RT (retreatment). Some retreatments are for second cancer and other for metastatic events. We have used the estimated rate of $52.3 \%$ for determining the optimal RT demand for the future year.

2.3 Evaluation of new radiotherapy centres based on efficiency, service availability and equity

We have used the proposed modelling work to estimate the change in patient access with the opening of new RT centre. We have employed three measures to quantitatively assess/evaluate the locations of new RT centres - general efficiency, service availability and 
equity. These measures have been used previously to evaluate locations of healthcare facilities (Batta et al. 2014, Burkey et al. 2012).

A. General efficiency: for general efficiency, we have considered the average distance between any potential cancer patient and his closest RT facility. In literature, various researchers have used such measures for evaluating facility location problems in healthcare. Batta et al. (2014) have used dispersion and population criteria as the proxies for measures of general efficiency. They focussed on minimising the cost of providing the service. We are using this measure as a proxy for general efficiency for RT service provision.

B. Service availability: The proxy for service availability is defined to be the population coverage for RT treatments. Specifically, the coverage can be measured as the proportion of the potential cancer patients that is located within a pre-specified distance from their closest RT facility. This measure of coverage was first defined by Toregas et al. (1971). According to this measure, a cancer patient is considered covered if he is living no farther than 50KMs from the closest facility. The union of coverage areas for each RT centre is then represents service availability. We are considering $50 \mathrm{KM}$ as the coverage driving distance because previous research studies (Gabriel et al. 2012) and our analysis of cancer treatment records indicated that patients living less than $50 \mathrm{Kms}$ from the closest RT facility were receiving highest rates of radiotherapy based treatments. Radiotherapy is considered a necessary component of treatment in 52\% of all newly diagnosed cancers (Delaney et al. 2005). Radiotherapy is considered to be most cost effective than surgery and chemotherapy, when all costs across the life cycle is considered (Ploquin and Dunscombe 2008).

C. Equity: the equity measure helps to quantify aspects related to fairness, justice of the service provision, and neediness. Burkey et al. (2012) have used a measure known as Gini index, which is based on Lorenz curve in economics. These curves are used as a graphical illustration of inequality for any quantitative study where inequality is measured. Gini index is a scalar measure based on Lorenz curve to quantify inequality (Gini 1921). Gini index is always between 0 (indicating total equality) and 1 (indicating total inequality). We have used this measure for determining the equality for potential cancer patients to access RT based treatment.

All of these measures are used to evaluate the alternative locations of new RT centres. All the methods in this section are coded in $\mathrm{R}$, which is an open source software environment for 
statistical computing and graphics using open source R libraries such as maptools, ggplot2, plyr, ineq, rgeos, rgdal, sp, RColorBrewer for this approach development and data analysis. Interested parties can access the software tools developed in this study freely by contacting authors.

\section{Results and Discussion}

This study estimates a spatially explicit demand and accessibility for radiotherapy services in the NSW state of Australia using methods described above. The choice of the NSW was justified by the accurate data currently available for NSW and a well-defined geographical area that is known to mirror average demographic distributions in Australia. Figure 2 illustrates population grid $\left(1 \mathrm{Km}^{2}\right)$ distributed in the whole of NSW state and existing locations of RT centres in NSW.

Non-linear regression for each age-sex group were conducted on the past cancer incidence data from AIHW. In both males and females, a statistically significant time trend was found for the following groups ( $\alpha<.05)$ : '40-44 yrs', '45-49 yrs', '50-54 yrs', '55-59 yrs', '60-64 yrs', '65-69 yrs', '70-74 yrs', '75-79 yrs', '80-84 yrs', and '85+ yrs'. Figure 3 illustrates the results from non-linear regression analysis (all age groups combined) of cancer incidences projected in future and its comparison with the past incidence rates. In Figure 3, the overall rates for cancer incidence in females are not increasing compared to the incidences in males. The dotted lines in Figure 3 represent the results of the model and the solid lines represent the actual rates observed. The results of the ASR prediction model for future years were then applied to the ABS population projections. 


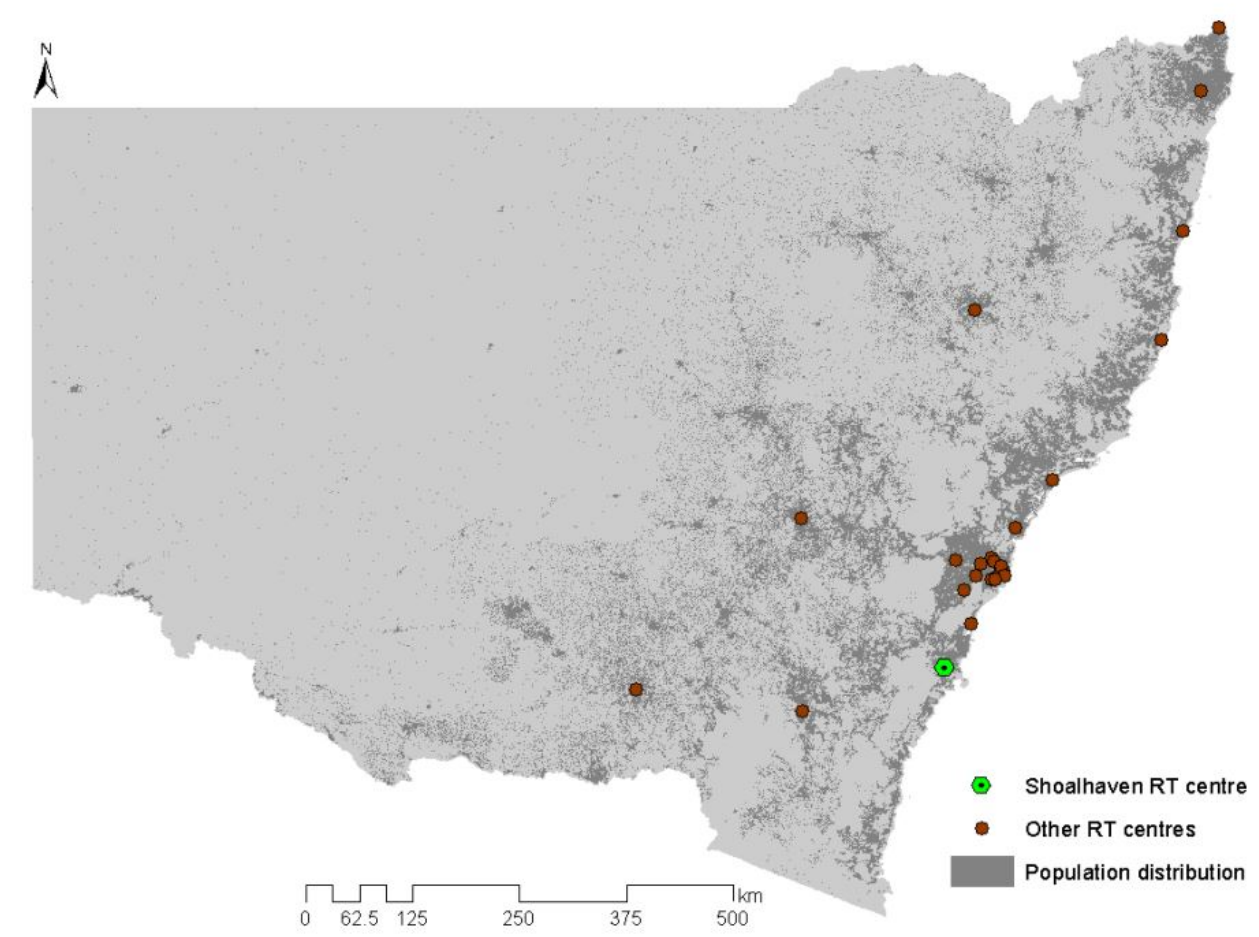

Figure 2: Distribution of population grid $\left(1 \mathrm{Km}^{2}\right)$ and radiotherapy centres in the NSW state

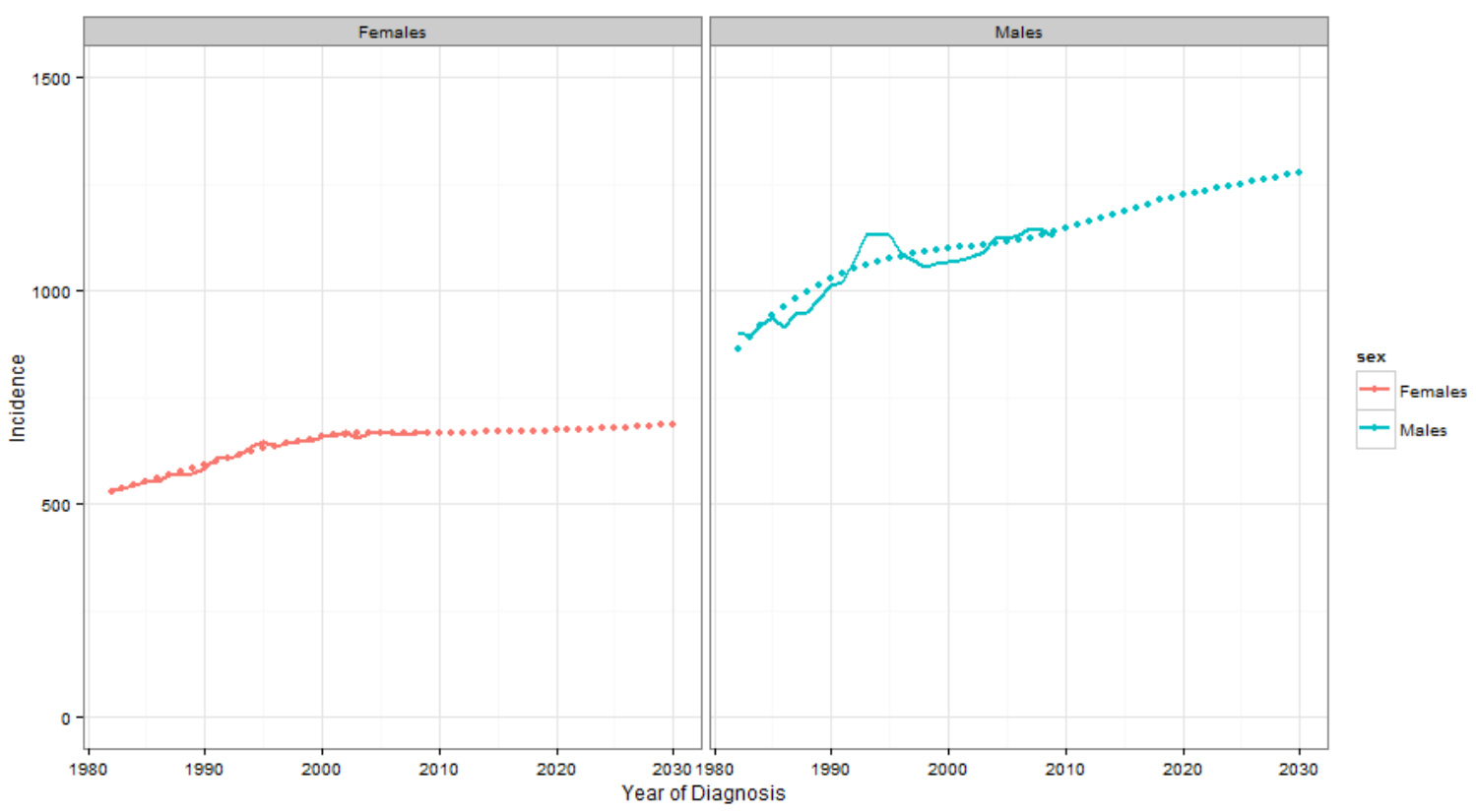

Figure 3: Predicted (points) and observed (solid line) incidence rates (per 100,000) for all cancers in males and females in Australia

The NSW population data provided by the ABS was used in this study. Figure 4 illustrates the growth in overall population of NSW from 2011 to 2026. It is evident that the population will grow significantly in future. However, the growth in population in different age groups and sex varies across NSW. 


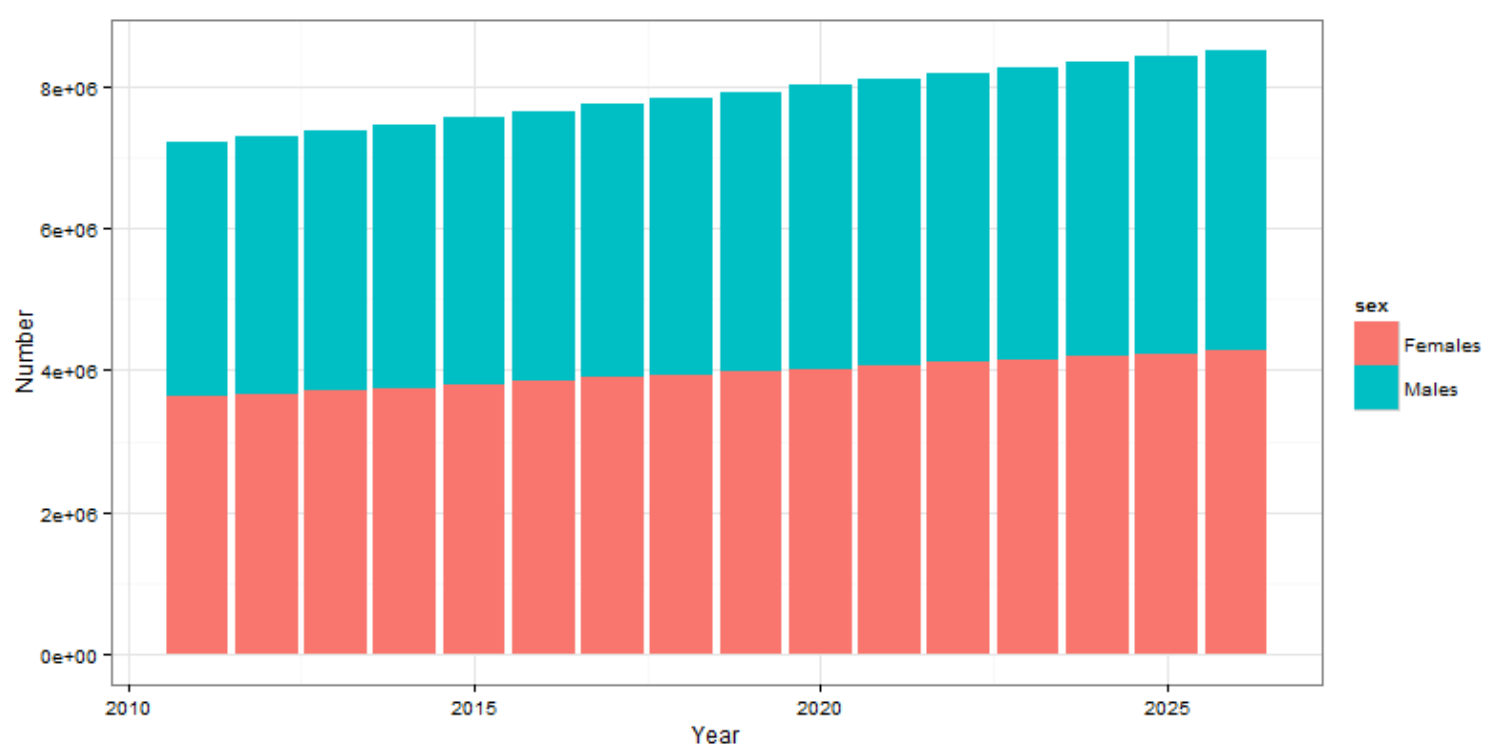

Figure 4: Projected population between 2011 and 2026 based on ABS population projections

Figure 5 illustrates the growth of population in different age-sex group in year 2011 and 2026. In year 2026, number of individuals in age groups with a higher cancer incidence (i.e., above 50 years) will be larger than 2011 for both females and males (see Figure 5). There will be more aged people living in year 2026 in NSW, and because of cancer incidence in the aged population sub-groups, more cancer cases.

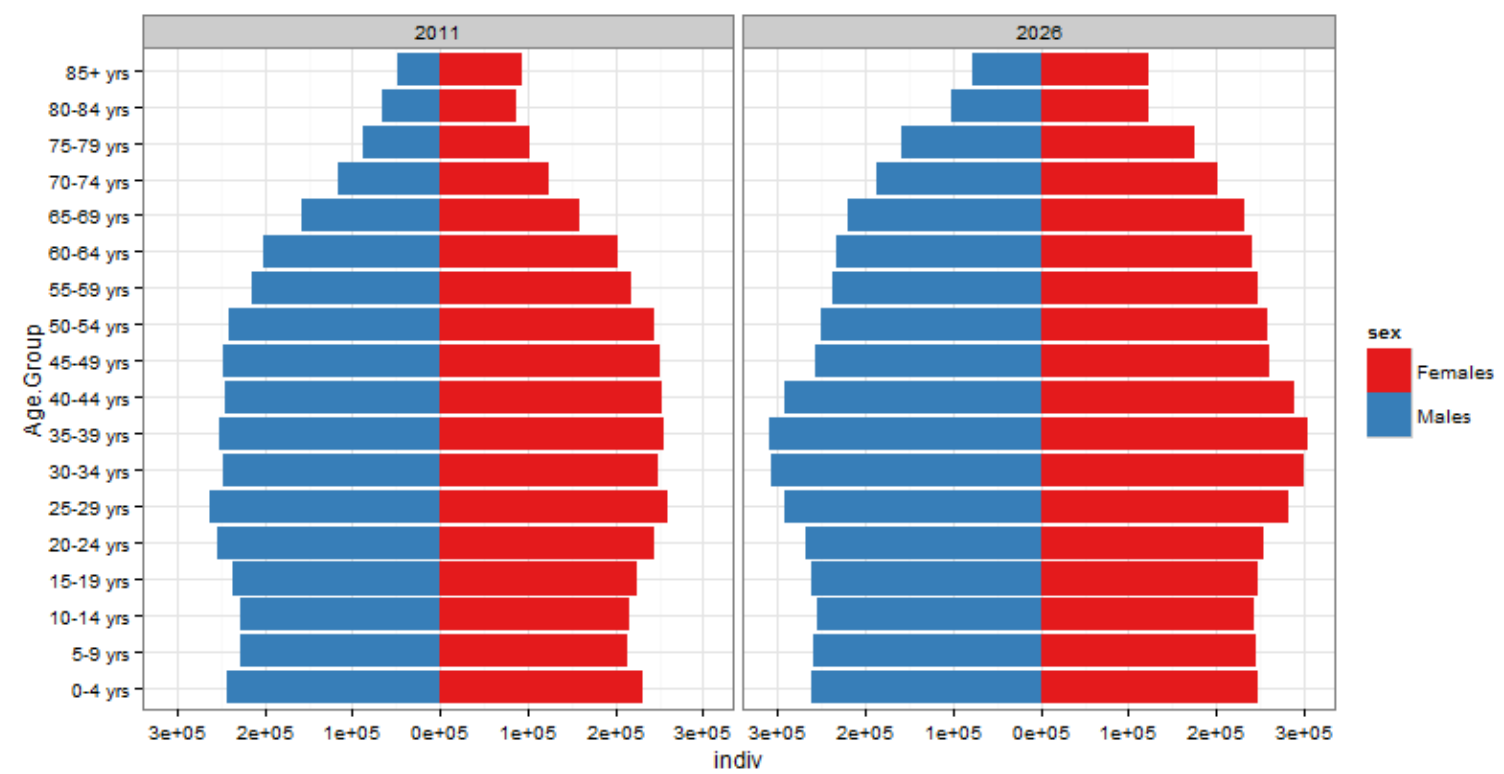

Figure 5: Age structure of NSW population in years 2011 and 2026

Figures $6 \mathrm{a}$ and $6 \mathrm{~b}$ illustrate the cancer incidences (cancer cases per 100,000 individuals) for year 2011 (Figure 6a) and future year 2026 (Figure 6b). The cancer incidences in all the local government area (LGA) of NSW (total of 154) are based on the age-sex specific rates obtained based on non-linear regression modelling (as discussed in Section 2.2.A) and future 
population estimates data (see Figure 5). In year 2011, the average cancer incidence at LGA level is approximately 612 per 100,000 individuals. However, it increases to 805 (approx.) per 100,000 individuals for future year 2026. Based on the spatial distribution of cancer incidences in year 2011 and 2026, which suggests more cancer cases in the coastal, mideastern and southern LGAs of NSW, planning of radiotherapy services for these areas are important.

(a)

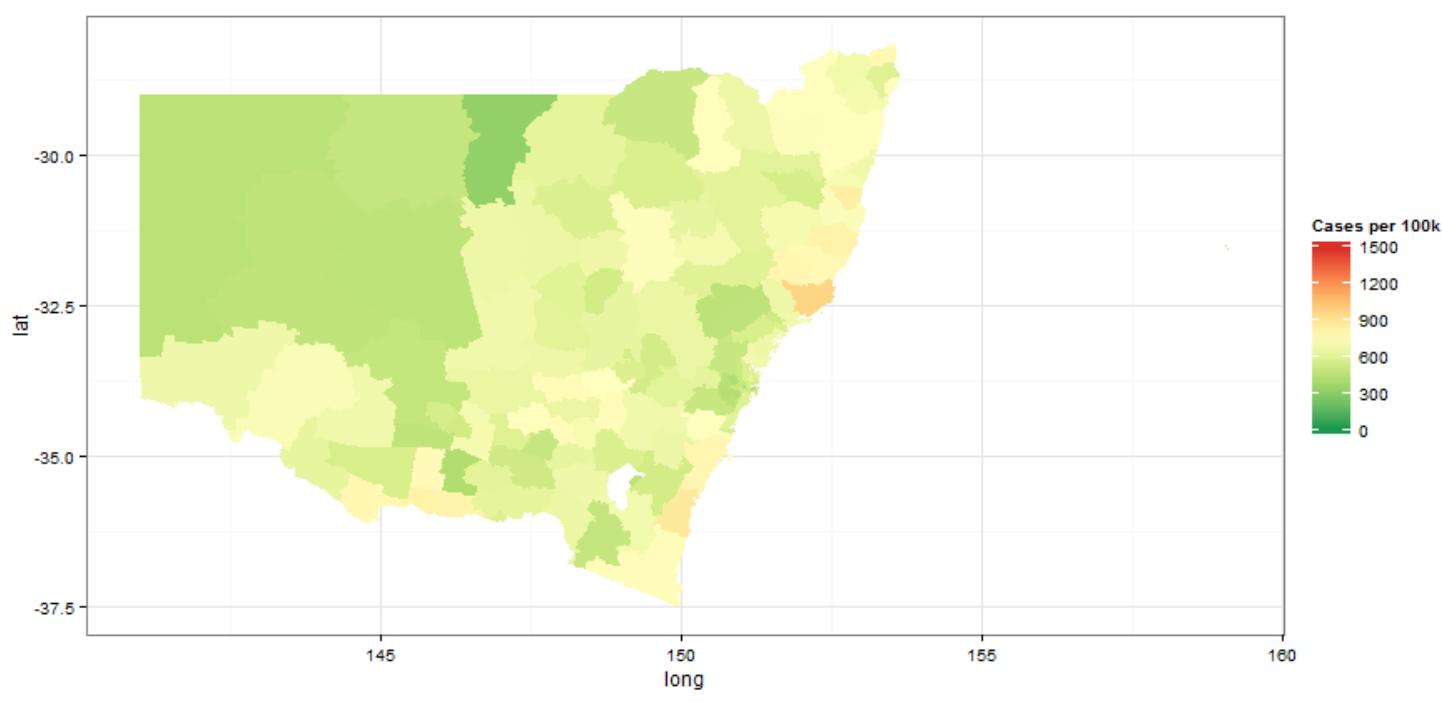

(b)

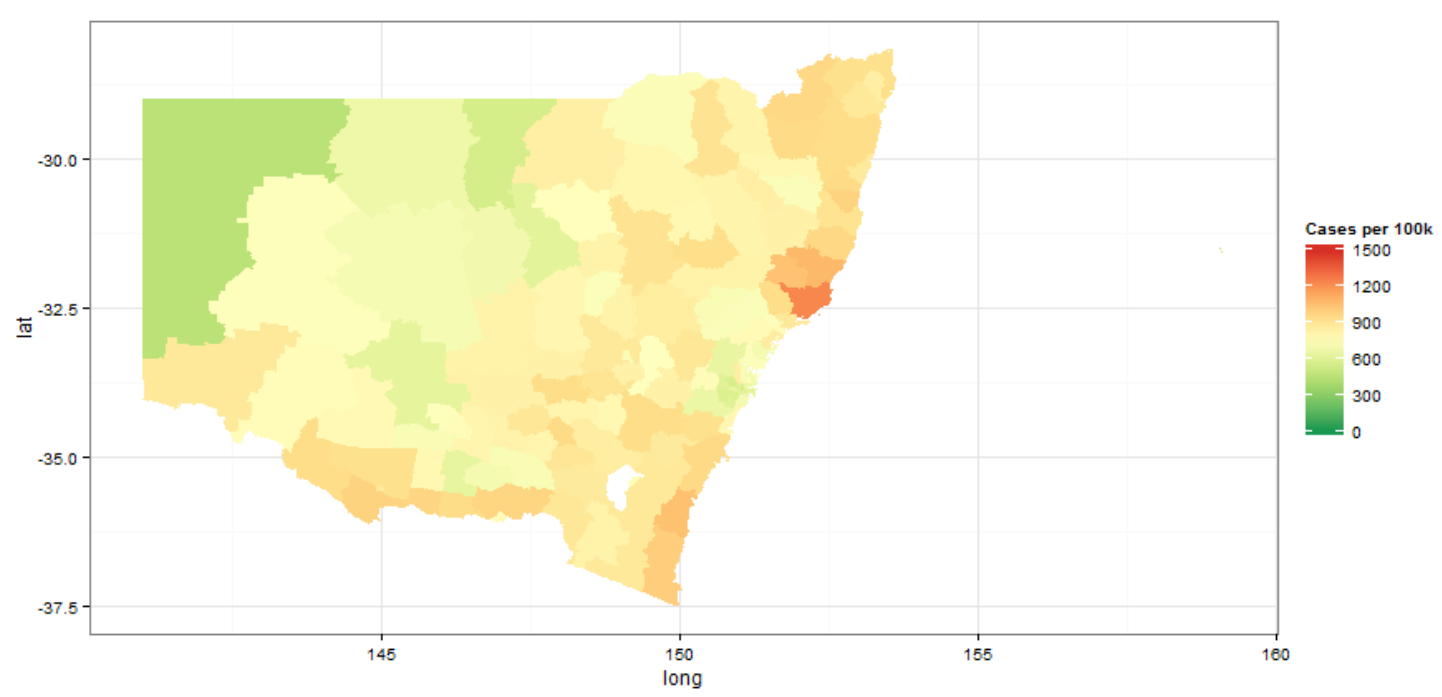

Figure 6: Overall cancer incidences in year 2011 (a) and 2026 (b) in NSW state of Australia

Figure 7 depicts driving distance polygons or the accessible regions considering all radiotherapy centres as trip origins. We have included existing radiotherapy centres in the NSW and also the nearby centres from the two bordering states- Victoria and Queensland. This was done to account for patient leakage in to those centres from NSW. As described in 
Section 2.2.C, for each LGA the fraction of population living in each distance band is estimated. Table 2 shows these calculated fractions for two LGAs as an example.

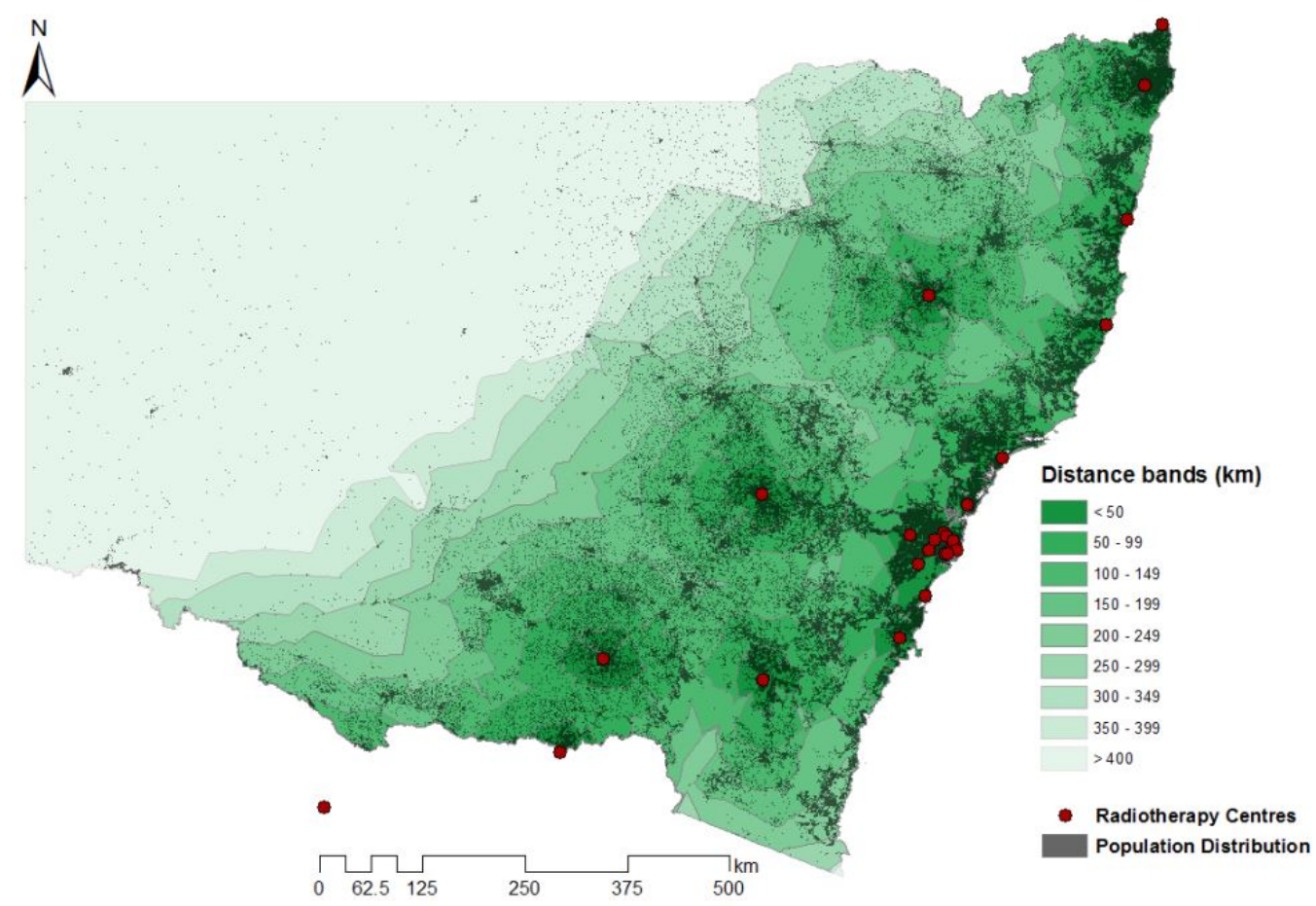

Figure 7: Constant driving distance polygons from radiotherapy centres

Table 2. Estimated fractions of population living in constant driving distance bands from radiotherapy centres

\begin{tabular}{l|l|l}
\hline \multicolumn{1}{c|}{ LGA } & \multicolumn{1}{c|}{ Distance band } & \multicolumn{1}{c}{$\begin{array}{c}\text { Fraction of } \\
\text { population }\left(F R A_{i, j}\right)\end{array}$} \\
\hline Hawkesbury & $<50$ & 0.88 \\
\hline Hawkesbury & $50-99$ & 0.11 \\
\hline Hawkesbury & $100-149$ & 0.01 \\
\hline Wingecarribee & $<50$ & 0.15 \\
\hline Wingecarribee & $50-99$ & 0.85 \\
\hline
\end{tabular}

The distance-based LGA ratios $\left(F R A_{i, j}\right)$ in the NSW (illustrated in Table 2) were used together with the cancer cases for respective LGA (illustrated in Figure 6) to estimate the total number of cancer patients residing in each distance band. The cancer cases residing in each distance band can be used for planning and locating new radiotherapy centres. 
Table 3 provides the comparison/validation of the proposed model with the actual cancer cases data from Cancer Institute NSW for a local health district (CI-NSW, 2014). The most up to date data available for comparison from Cancer Institute NSW is from 2004-2008 and therefore it is used in this study for comparison purposes. Average actual cancer counts per year shows that the proposed result for year 2011 matches closely. It can be seen that the predicted results are on slightly high compared to actual numbers. This is due to the fact that the predictions were made for the year 2011-2015, and it is compared against actual cancer counts from year 2004-2008.

Table 3. Comparison between actual cancer incidence dataset and predicted results

\begin{tabular}{l|c|c|c|c|}
\hline $\begin{array}{l}\text { Local Government } \\
\text { Area }\end{array}$ & $\begin{array}{c}\text { Actual Cancer Count } \\
\text { (2004- 2008) }\end{array}$ & $\begin{array}{c}\text { Average Actual } \\
\text { Cancer } \\
\text { Count/year }\end{array}$ & $\begin{array}{c}\text { Predicted Count } \\
\text { (2011) }\end{array}$ & $\begin{array}{c}\text { Predicted Count } \\
(2011-2015)\end{array}$ \\
\hline Kiama & 627 & 125 & 150 & 815 \\
Shellharbour & 1,475 & 295 & 369 & 1,987 \\
Shoalhaven & 3,481 & 696 & 765 & 3,987 \\
Wollongong & 5,223 & 1,045 & 1,224 & 6,482 \\
NSW & $\mathbf{1 7 7 , 5 1 9}$ & $\mathbf{3 3 , 5 0 4}$ & $\mathbf{4 1 , 2 2 9}$ & $\mathbf{2 1 8 , 7 0 0}$ \\
\hline
\end{tabular}

The proposed approach was then applied to estimate change in access of cancer patients with the opening of new radiotherapy centre in Shoalhaven LGA (see Figure 3). We have run the travel distance modelling method for the scenarios before and after opening of new RT centre. The change in cancer patient's access for before and after opening of RT centre is presented in Figure 8. It can be seen that there are more than 600 cancer cases residing in the $50 \mathrm{~km}$ road distance from the nearest RT centre when the new RT centre is operational. The number of RT cases residing within $50 \mathrm{~km}$ of nearest RT centre in the Illawarra and Shoalhaven local health district before and after is 1120 and 1511. Interestingly, within 6 months of opening the Shoalhaven RT service is already managing 500 new RT cases per month (A.Miller, personal communication). Due to the opening of new centre, there are less cancer cases living in distance bands (in km) 50-99, 100-149, 150-199, 200-249, therefore the difference between the numbers of cancer cases is negative. For larger distance bands (250299, 300-349, 350-399, 400+), the number of cancer cases are constant for before and after RT centre placement. 


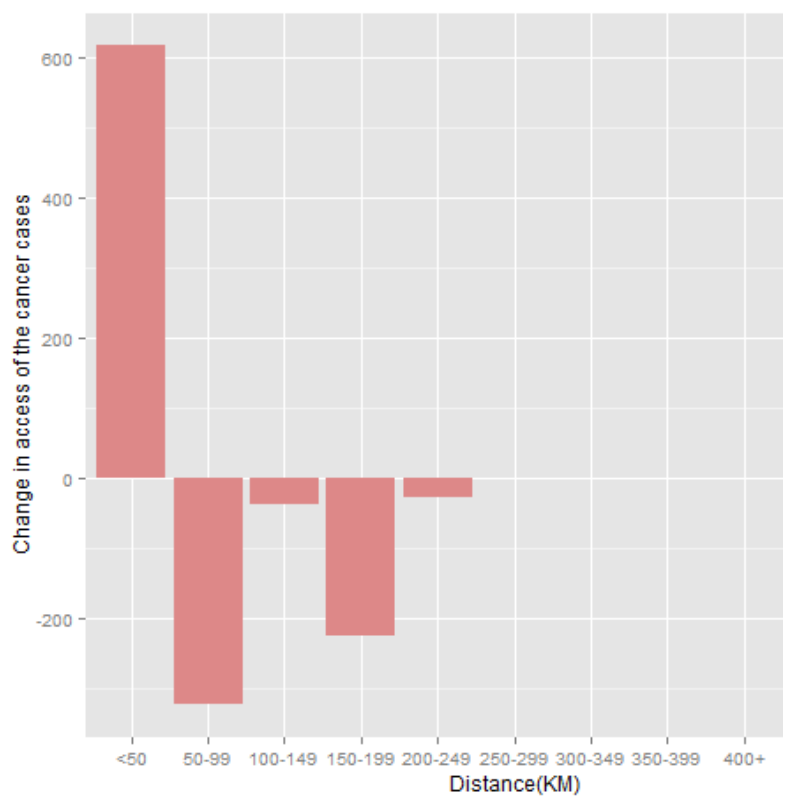

Figure 8: Change in cancer cases access before and after opening of new RT centre in Shoalhaven

To further illustrate the applicability of proposed approach, we have compared different scenarios of placement of RT units in NSW. For this, we considered two potential locations in NSW to place new RT facility - (i) Dubbo; and (ii) Moruya (see Fig. 9). These scenarios are then compared in terms of general efficiency; service accessibility and RT service equality (see Section 2.3). The results of the comparison are presented in Table 4.

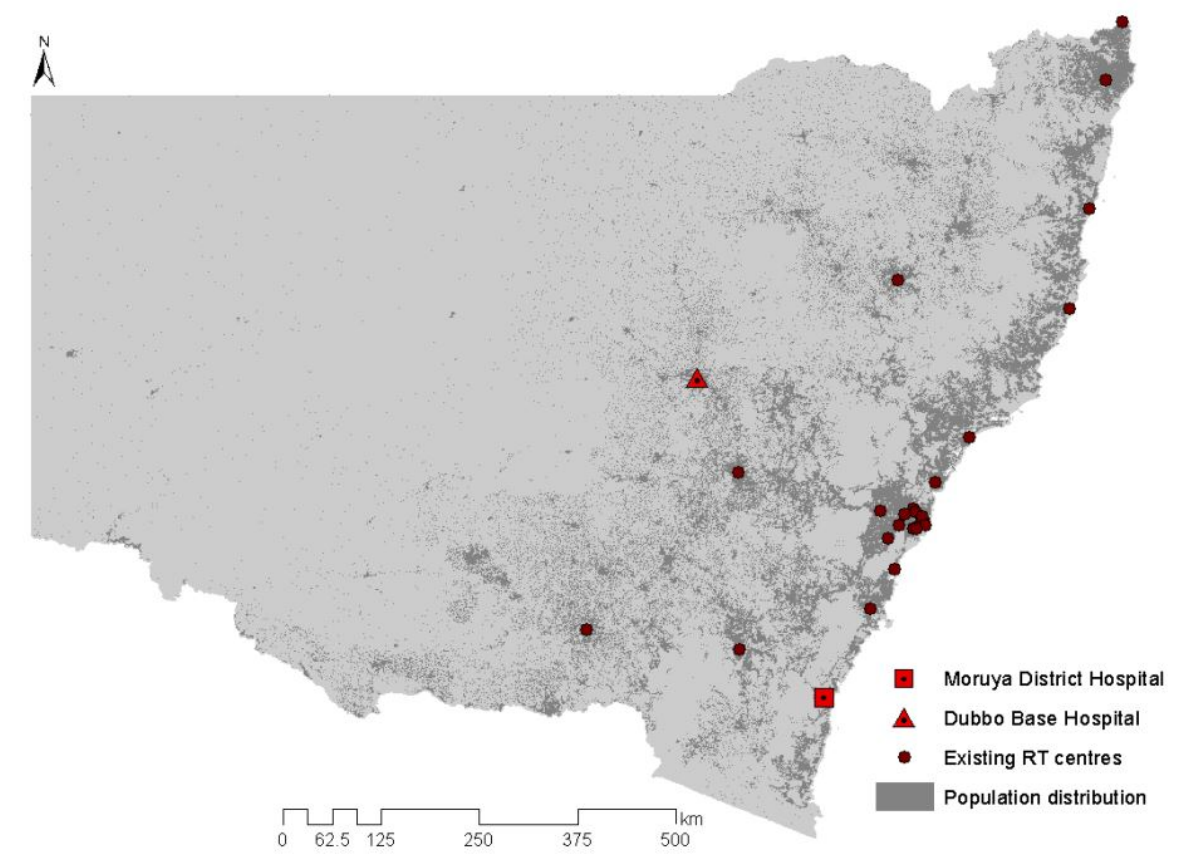

Figure 9: Potential locations for new RT centre used for scenario analysis 
Table 4 shows that opening a new RT centre at Dubbo or Moruya offers advantages in terms of accessibility to cancer patients. Scenario - Dubbo presents better opportunity for general efficiency improvement (i.e. 5.19\% more than the baseline), service availability improvement ( $0.71 \%$ more); and, service equality increases by $1.51 \%$ compared to baseline. However, for the case where new RT facility has to be opened in Moruya, the general efficiency and service availability increases by $3.88 \%$ and $0.59 \%$, but the distance based equality for RT services decreases by $0.47 \%$. This implies that opening new RT centre in Dubbo offers better accessibility measures compared to Moruya. It should be noted that the Gini index (inequality) measure presented in Table 4 should be minimised.

Table 4: Comparison of scenarios based on general efficiency, service accessibility and service equality

\begin{tabular}{lccc}
\hline & General Efficiency & Service availability & $\begin{array}{c}\text { Gini Index } \\
\text { (Inequality) }\end{array}$ \\
\hline Scenario - Dubbo & $27.88(5.19 \%$ more $)$ & $0.865(0.71 \%$ more $)$ & $0.408(1.51 \%$ more $)$ \\
Scenario - Moruya & $28.27(3.88 \%$ more $)$ & $0.864(0.59 \%$ more $)$ & $0.416(0.47 \%$ less $)$ \\
Baseline & 29.41 & 0.859 & 0.414 \\
\hline
\end{tabular}

Table 5 illustrates the demand for the RT services based on the radiotherapy rates estimated in Section 2.2.D. RT demand at the current service level is calculated by applying RT rates by road distance (estimated in Table 1) to the number of cancer patients living in each distance bands (see Section 2.2.B and 2.2.C). However, the optimal RT demand is estimated by using cancer incidence number and optimal RT utilisation rates (52.3\%, see Section 2.2.D).

Table 5: RT demand from year 2011 to 2026

\begin{tabular}{|ccc}
\hline Year & $\begin{array}{c}\text { RT Demand at current } \\
\text { service level }\end{array}$ & $\begin{array}{c}\text { Optimal RT Demand } \\
\text { based on evidence }\end{array}$ \\
\hline $\mathbf{2 0 1 1}$ & 13506 & 17614 \\
$\mathbf{2 0 1 2}$ & 13905 & 18144 \\
$\mathbf{2 0 1 3}$ & 14319 & 18680 \\
$\mathbf{2 0 1 4}$ & 14736 & 19230 \\
$\mathbf{2 0 1 5}$ & 15180 & 19796 \\
$\mathbf{2 0 1 6}$ & 15629 & 20370 \\
\hline $\mathbf{2 0 1 7}$ & 16088 & 20956 \\
$\mathbf{2 0 1 8}$ & 16536 & 21560 \\
$\mathbf{2 0 1 9}$ & 16968 & 22149 \\
\hline $\mathbf{2 0 2 0}$ & 17395 & 22749 \\
\hline $\mathbf{2 0 2 1}$ & 17810 & 23378 \\
\hline $\mathbf{2 0 2 2}$ & 18255 & 24015 \\
\hline $\mathbf{2 0 2 3}$ & 18708 & 24652 \\
\hline
\end{tabular}




\begin{tabular}{lll}
\hline $\mathbf{2 0 2 4}$ & 19156 & 25285 \\
$\mathbf{2 0 2 5}$ & 19612 & 25917 \\
$\mathbf{2 0 2 6}$ & 20070 & 26545 \\
\hline
\end{tabular}

It can be concluded from Table 5 that supply of RT services significantly lags behind the evidence-based optimal demand and new RT centres should be established to deal with such shortfall. This trend of shortfall will continue in future at the current service levels. The proposed approach offers an innovative way to assess/evaluate the alternative locations of new RT services based on - general efficiency, service availability, and equality.

\section{Conclusion}

The treatment of cancer, which touches a large proportion of the community, represents a significant health and economic burden in Australia. Nearly one in two men and one in three women have a lifetime risk of being diagnosed with cancer in NSW. Radiotherapy (RT) based cancer treatments are often integral to achieving permanent or long-term remission. There will be large demand for RT services for cancer patients in NSW in next ten years. However, decision on new linear accelerator (LINAC) units take time, resulting in local congestion of services or unreasonable travel time for patients, with some individuals opting out of RT treatment reducing demand but not the need. Thus, there is pressing need to rationally justify the placement of new radiotherapy centres to effectively meet the future RT need of future cancer patients. This study makes a unique contribution to the policy debate on placement of RT treatment centres through the establishment of an integrated modelling approach. This study marks an attempt at informing the government about the best placement locations for RT centres to maximise the RT service capacity to meet future cancer patient demand.

The demonstrated generic approach includes cancer incidence predictive modelling to estimate the cancer cases residing within different travel distance bands from the RT centres, and is easily applied to the NSW circumstance. The methods described in this research study can be applied in other jurisdictions with availability of the necessary data.

We are aware of limitations in our current approach which are being addressed with more research. We have assumed that the OSM road network data is complete. As the OSM data becomes more complete in time, we only need to reimport that dataset to update the analysis. 
In estimating future cancer cases, we assume that the residential locations remain the same, but including research on dynamic land use modelling is expected to better account for residential area growth. The cancer incidence modelling assumes the same incidence across different local government areas (LGAs) and that the past trends will continue in future. Lastly, the model can be refined to include detailed information about the individual cancer incidences, radiotherapy demand, and accessibility profiles rather than using all inclusive assessments. In current study, authors are dealing with all types of cancer and their aggregated rates; therefore, survival analysis is not conducted for different types of cancer cases and their stages. We hope this type of analysis can be conducted in future based on our work.

\section{References}

AIHW (2014), Cancer Data, (Accessed Jun 2014) http://www.aihw.gov.au/cancer-data/

Asthana S, Gibson A, Moon G, Brigham P. Allocating resources for health and social care: the significance of rurality. Health Society of Care Community 2003; 11(6): 486-493.

Athas WF, Adams-Cameron M, Hunt WC, Amir-Fazli A, Key CR. Travel distance to radiation therapy and receipt of radiotherapy following breast-conserving surgery. $J$ Natl Cancer Inst, 2000, 92, 269-71.

Baade, P. D., Dasgupta, P., Aitken, J. F. \&Turrell, G. Distance to the closest radiotherapy facility and survival after a diagnosis of rectal cancer in Queensland. Med J Aust, (2011), 195, 350-354.

Barton M, Delaney G. Decade of investment in radiotherapy in New South Wales: why does the gap between optimal and actual persists? Journal of Medical Imaging and Radiation Oncology. Volume 55, Issue 4, August 2011, pp: 433-441

Barton M. Radiotherapy utilization in New South Wales from 1996 to 1998.AustralasRadiol 2000; 44: 308-14.

Batta, R, Lejeune, M, Prasad, S, Public facility location using dispersion, population, and equity criteria, European Journal of Operational Research, Volume 234, Issue 3, 1 May 2014, Pages 819-829.

Bennett J (2010), OpenStreetMap, Packt Publishing, Birmingham, GBR. p. 252. 
Burkey, M.L., Bhadury, J., Eiselt, H.A., A location-based comparison of health care services in four U.S. states with efficiency and equity, Socio-Economic Planning Sciences, Volume 46, Issue 2, June 2012, Pages 157-163.

Cancer Care Ontario Radiation Treatment Utilization - CSQI10.at $<$ https://www.cancercare.on.ca/cms/one.aspx?pageId=14959>

Cancer Council Australia.Facts and Figures. Sydney: Cancer Council Australia (2012)

CI-NSW (Cancer Institute NSW), Online statistics module, Accessed: 13 Nov. 2014 http://www.cancerinstitute.org.au/data-and-statistics/cancer-statistics/online-statistics$\underline{\text { module }}$

Coory, M.D., Ho, T. and Jordan, S. J., Australia is continuing to make progress against cancer, but the regional and remote disadvantage remains, Med J Aust 2013; 199 (9): 605608.

Delaney G "Radiotherapy in Cancer Care: Estimating the Optimal Utilization from a review of Evidence-Based Clinical Guidelines” PhD Thesis, 2007.

Delaney G., Jacob S., Featherstone C., Barton B, (2005) "The Role of Radiotherapy in Cancer Treatment: Estimating Optimal Utilization from a Review of Evidence-Based Clinical Guidelines” Cancer, Vol. 104, No. 6, pp. 1129-1137.

Delaney GP, Jacob S., Featherstone C., Barton MB. Radiotherapy in Cancer Care: Estimating Optimal Utilisation from a Review of Evidence-Based Clinical Guidelines. Collaboration for Cancer Outcomes Research and Evaluation (CCORE), Liverpool Hospital, Sydney, Australia, 2003.

Delaney, G., Jacob, S., Featherstone, C. \& Barton, M. The Role of Radiotherapy in Cancer Treatment: Estimating Optimal Utilization from a Review of Evidence-Based Clinical Guidelines. Cancer 104, 1129-1137 (2005).

Denham JW. How do we bring an acceptable level of radiotherapy services to a dispersed population? Australas Radiol 1995; 39: 171-3.

Denham JW. How do we bring an acceptable level of radiotherapy services to a dispersed population? Australas Radiol 1995; 39: 171-3.

DoH (2013), Statistical Local Area Population Projections, 2011 (base) to 2026, Preliminary, (Accessed, Jun 2014): http://www.health.gov.au/internet/main/publishing.nsf/Content/ageing-stats-lapp.htm

DoH (2014), Radiation Oncology and Radiotherapy Services, (Accessed, Jun 2014): http://www.health.gov.au/internet/main/publishing.nsf/Content/health-roi-radiotherindex.htm 
Douglas, K, Douglas, S (2005), PostgreSQL, SAMS Technical Publishing, Indianapolis, IN, p. 1032.

Einhorn J, Frödin J-E, Möller T et al . Radiotherapy practices in Sweden compared to the scientific evidence. Acta Oncol 1996; 35 (Suppl. 6): 89-97

Gabriel, G., Barton, M., Delaney, G. Radiotherapy utilisation rates in NSW and ACT [200406], a data linkage and GIS experience. Innovation in Cancer Treatment and Care NSW Conference, 2012.

Gini, C., Measurement of inequality of incomes, The Economic Journal, 31 (121) (1921), pp. $124-126$

Gray, J (2008), Getting Started with Quantum GIS. LINUX Journal, (Accessed, August 2014): http://www.linuxjournal.com/content/getting-started-quantum-gis

Greenberg ER, Chute CG, Stukel T, et al. Social and economic factors in the choice of lung cancer treatment.A population-based study in two rural areas. New Engl $\mathrm{J}$ Med 1988;318:612-7.

Haklay, M (2010), How good is volunteered geographical information? A comparative study of OpenStreetMap and ordnance survey datasets, Environment and Planning B: Planning and Design, Vol. 37, No. 4, pp. 682-703.

Mackillop WJ, Groome PA, Zhang-Solomons J et al. Does a centralized radiotherapy system provide adequate access to care? J ClinOncol 1997; 15 : 1261-71.

Madelaine J, Guizard AV, Lefevre H, Lecarpentier MM, Launoy G. Diagnosis, treatment and prognosis of lung cancer in the Manche (France) (1997 - 1999) according to patient socioeconomic characteristics, Review Epidemiol. Sante Publique 2002;50:383-92.

Morgan G. Why has Radiotherapy Utilisation not improved since 1999? Journal of Medical Imaging and Radiation Oncology. 2011 August; Volume 55 (Number 4) p347-350

Morgan, G. W. et al. 'GAP' in radiotherapy services in Australia and New Zealand in 2009. Journal of Medical Imaging and Radiation Oncology, 54, 287-297 (2010).

Payne S, Jarrett N, Jeffs D. The impact of travel on cancer patients' experiences of treatment: a literature review. European Journal of Cancer Care 2000; 9(4): 197-203.

pgRouting Contributors (2013), pgRouting Manual, Release 2.0.0, p. 88, (Accessed, August 2014): http://docs.pgrouting.org/2.0/en/pgRoutingDocumentation.pdf

Ploquin, N. P., and Dunscombe, P. B., 2008. The cost of radiation therapy. Radiotherapy Oncology, 86 (2): 217-223. 
Punglia RS, Weeks JC, Neville BA, Earle CC. (2006), Effect of distance to radiation treatment facility on use of radiation therapy after mastectomy in elderly women. Int $J$ Rad Oncol Bio Phys 2006; 66:56-63.

Ragaz J, Jackson SM, Le N, Plenderleith IH, Spinelli JJ, Basco VE, Wilson KS, Knowling MA, Coppin CM, Paradis M, Coldman AJ, Olivotto IA. Adjuvant radiotherapy and chemotherapy in node-positive premenopausal women with breast cancer. N Engl J Med. 1997 Oct 2;337(14):956-62.

ROTC (Radiation Oncology Tripartite Committee), (2012a), Planning For The Best: Tripartite National Strategic Plan for Radiation Oncology 2012 - 2022, The Royal Australian and New Zealand College of Radiologists

ROTC (Radiation Oncology Tripartite Committee), (2012b),Projecting the radiation oncology workforce, Input to the Tripartite National Strategic Plan For Radiation Oncology in Australia, May 2012, The Royal Australian and New Zealand College of Radiologists

Schroen, A. T., Brenin, D. R., Kelly, M. D., Knaus, W. A. \& Slingluff Jr, C. L. Impact of patient distance to radiation therapy on mastectomy use in early-stage breast cancer patients. Journal of Clinical Oncology 23, 7074-7080 (2005).

Shukla, N, Keast J.E., Ceglarek, D. (2014) "Modelling variations in hospital service delivery based on real time locating information" Applied Mathematical Modelling, Volume 38, Issue 3, Pages 878-893

Shukla, N., Keast,J.E., Ceglarek, D. (2014) "Improved workflow modelling using role activity diagram-based modelling with application to a radiology service case study" Computer Methods and Programs in Biomedicine, Volume 116, Issue 3, Pages 274-298.

SSDB (Statewide Services Development Branch, 2004). Radiotherapy management information system report 2003. Sydney: NSW Health Department, 2004

The Allen Consulting Group Projecting the radiation oncology workforce. Input to the Tripartite National Strategic Plan For Radiation Oncology in Australia. 1-41 (RANZCR: 2012).

Toregas, C., Swain, C., ReVelle, C., Bergman, L., The location of emergency service facilities, Operations Research, 19 (1971), pp. 1363-1373

Tyagi, S., Choudhary, A., Cai, X., Yang, K., (2015) "Value stream mapping to reduce the lead-time of a product development process" International Journal of Production Economics, Volume 160, February 2015, Pages 202-212 
Vinh-Hung, V., Verschraegen, C., "Breast-Conserving Surgery With or Without Radiotherapy: Pooled-Analysis for Risks of Ipsilateral Breast Tumor Recurrence and Mortality” Journal of the National Cancer Institute, Vol. 96, No. 2, 115-21.

Wigg, D. R. \& Morgan, G. W. Radiation oncology in Australia: workforce, workloads and equipment 1986-1999. Australasian Radiology 45, 146-169 (2001).

Wilcox, S. W., Aherne, N. J., McLachlan, C. S., McKay, M. J., Last, A. J. and Shakespeare, T. P. (2015), Is modern external beam radiotherapy with androgen deprivation therapy still a viable alternative for prostate cancer in an era of robotic surgery and brachytherapy: A comparison of Australian series. Journal of Medical Imaging and Radiation Oncology, 59: 125-133.

Williams, M. V. Regional inequalities and cancer. BMJ 339, (2009).

Yu, X.Q., Luo, Q., Smith, D. P., O’Connell, D.L. and Baade, P.D., Geographic variation in prostate cancer survival in New South Wales, Med J Aust 2014; 200 (10): 586-590.

Zhan, F.B. and Noon, C.E.. Shortest Path Algorithms: An Evaluation Using Real Road Networks. Transportation Science, 32 (1) (1998), pp. 65-73 